\title{
Identification of Key Genes and Candidated Pathways in Human Autosomal Dominant Polycystic Kidney Disease by Bioinformatics Analysis
}

\author{
Dongmei Liu ${ }^{a}$ Yongbao Huo ${ }^{b}$ Sixiu Chen ${ }^{a}$ Dechao Xua Bo Yang ${ }^{a}$ \\ Cheng Xue ${ }^{a} \quad$ Lili Fu $^{a}$ Lei Bu ${ }^{a}$ Shuwei Song ${ }^{a} \quad$ Changlin Mei ${ }^{a}$ \\ a Kidney Institute, Department of Nephrology, Changzheng Hospital, Second Military \\ Medical University, Shanghai, China; ${ }^{b}$ Department of Rheumatology and Immunology, \\ Changzheng Hospital, Second Military Medical University, Shanghai, China
}

\section{Keywords}

Autosomal dominant polycystic kidney disease - Bioinformatics analysis · Key genes .

Metabolic pathways

\begin{abstract}
Background/Aims: Autosomal dominant polycystic kidney disease (ADPKD) is the most common genetic form of kidney disease. High-throughput microarray analysis has been applied for elucidating key genes and pathways associated with ADPKD. Most genetic profiling data from ADPKD patients have been uploaded to public databases but not thoroughly analyzed. This study integrated 2 human microarray profile datasets to elucidate the potential pathways and protein-protein interactions (PPIs) involved in ADPKD via bioinformatics analysis in order to identify possible therapeutic targets. Methods: The kidney tissue microarray data of ADPKD patients and normal individuals were searched and obtained from NCBI Gene Expression Omnibus. Differentially expressed genes (DEGs) were identified, and enriched pathways and central node genes were elucidated using related websites and software according to bioinformatics analysis protocols. Seven DEGs were validated between polycystic kidney disease and control kidney samples by quantitative real-time polymerase chain reaction. $\boldsymbol{R e}$ sults: Two original human microarray datasets, GSE7869 and GSE35831, were integrated and thoroughly analyzed. In total, 6,422 and 1,152 DEGs were extracted from GSE7869 and GSE35831, respectively, and of these, 561 DEGs were consistent between the databases (291
\end{abstract}

D.L., Y.H., and S.C. contributed equally to this article. 


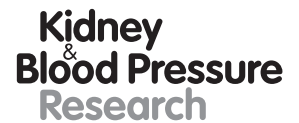

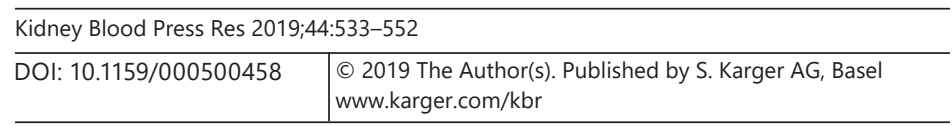

Liu et al.: Bioinformatics Analysis of ADPKD

upregulated genes and 270 downregulated genes). From 421 nodes, 34 central node genes were obtained from a PPI network complex of DEGs. Two significant modules were selected from the PPI network complex by using Cytotype MCODE. Most of the identified genes are involved in protein binding, extracellular region or space, platelet degranulation, mitochondrion, and metabolic pathways. Conclusions: The DEGs and related enriched pathways in ADPKD identified through this integrated bioinformatics analysis provide insights into the molecular mechanisms of ADPKD and potential therapeutic strategies. Specifically, abnormal decorin expression in different stages of ADPKD may represent a new therapeutic target in ADPKD, and regulation of metabolism and mitochondrial function in ADPKD may become a focus of future research.

(C) 2019 The Author(s)

Published by S. Karger AG, Basel

\section{Introduction}

Autosomal dominant polycystic kidney disease (ADPKD) is the most common genetic disorder of the kidney and is characterized by renal cyst formation [1]. ADPKD affects 12.5 million people worldwide among all ethnic groups and is the fourth leading cause of endstage renal disease (ESRD) requiring renal replacement therapy worldwide [2]. In China alone, there are an estimated 1.5 million patients with ADPKD [3], and 4.7\% of ESRD cases in Shanghai are attributed to ADPKD [4].

ADPKD is commonly caused by mutations in 2 genes, the polycystic kidney disease 1 (PKD1) [5] and 2 (PKD2) [6] genes. Functional loss of polycystin-1 or polycystin-2, the gene products of $P k d 1$ and $P k d 2$, respectively, leads to cyst initiation and expansion, through abnormalities in a variety of intracellular signaling pathways $[7,8]$. During the past 40 years, great advances have been made in understanding how ADPKD develops and how it can be diagnosed [9] and treated [10-13]. Notably, ADPKD is a common inherited disorder [14], and genetic mutations are central to exploring the underlying mechanisms of the disease.

High-throughput microarray analysis is a valid method for gene expression profiling that has been applied for elucidating key gene pathways associated with ADPKD. In the past 20 years, such studies have revealed many differentially expressed genes (DEGs) and affected signaling pathways in a PKD mouse model [15-20] as well as in ADPKD patients [21, 22]. However, ADPKD is still considered an incurable disease, and knowledge of the pathways involved and its pathogenesis remains incomplete $[1,10,11]$. Most genetic profiling data from ADPKD patients have been uploaded to public databases but not thoroughly analyzed. The data from independent studies are also limited by their source being a single cohort study and by the heterogeneity of samples. Thus, reanalyzing these data with an integrated bioinformatics approach might provide new insights for further research related to novel pharmacological interventions for patients with ADPKD.

One cross-species meta-analysis of conserved biological pathways in ADPKD has been reported [23], and the findings were valuable for further exploration of the potential of combination therapy in ADPKD. However, only a biological analysis was performed, and validation of corresponding genes was not carried out.

In the present study, we accessed 2 original human microarray datasets, GSE7869 [21] and GSE35831 [22], from the NCBI-Gene Expression Omnibus database (NCBI-GEO). Following a previously reported procedure for bioinformatics analysis [24, 25], we extracted DEGs in ADPKD from GSE7869 and GSE35831, separately, and then from among DEGs identified in both datasets, we obtained central node genes from the protein-protein interaction (PPI) network. We then validated the differential expression of identified genes by quantitative 


\section{Kidney \\ Blood Pressure \\ Research}

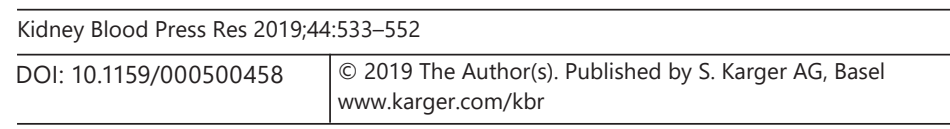

Liu et al.: Bioinformatics Analysis of ADPKD

polymerase chain reaction (qPCR) in a Pkd1 mouse model and ADPKD patient samples. Further analysis of the datasets provided insight into central node genes and enriched pathways in human ADPKD cases. These findings can improve our understanding of the molecular mechanisms of ADPKD with the potential for application in future biological research. Furthermore, these key genes and pathways might be employed as therapeutic targets for ADPKD.

\section{Materials and Methods}

Identification of Microarray Data and DEGs

The NCBI-GEO datasets were searched using the term "PKD," and 6 PKD mouse model datasets, 7 PKD rat datasets, and 2 ADPKD human datasets were retrieved. The 2 original human microarray datasets, GSE7869 and GSE35831, were downloaded. The microarray data of GSE7869 were based on the GPL570 platform (Affymetrix Human Genome U133 Plus 2.0 Array), and the kidney samples included 5 polycystic kidney specimens resected for medical reasons and noncancerous renal tissue from 3 nephrectomized kidneys with isolated renal cell carcinoma was used as normal control tissue in Canada [21]. The microarray data of GSE35831 [22] were based on the GPL6244 platform (Human Gene 1.0 ST Array), and the human renal tissue samples were acquired from one normal healthy individuals and 2 ADPKD patients in Korea.

The original high-throughput functional genomic expression data were obtained from PubMed as described by Shatakshee Chatterjee work group [23], which was processed in the TXT format. DEGs were identified using $t$ tests, and the cutoff criteria were defined as $p<0.05$ and $[\operatorname{logFC}]>1$ [26]. Consistently expressed DEGs in the 2 datasets were acquired by Funrich_V3 [27, 28].

\section{Gene Ontology and Pathway Enrichment Analysis}

The candidate DEGs were analyzed in terms of the functions and pathways showing enrichment using DAVID 6.7 [26]. Analysis of gene ontology (GO) and KEGG pathway was carried out [29, 30].

Analysis of PPI Network and Modules and Identification of Key Genes and Pathways

Proteins encoded by DEGs were input into STRING online, and a PPI network was obtained in TSV format [25]. Cytoscape software [31] and relative plugins [32] were utilized to process the PPI network. Node degree was calculated with Cytohubba plugins, and hub genes were filtered according to their numbers in the PPI network. Proteins in central nodes were considered as core proteins and key candidate genes that have important physiological regulatory functions.

Validation of Bioinformatics Analysis Result by Quantitative Real-Time PCR

Fluorescence qPCR was carried out to confirm the significantly altered expression of DEGs to verify the reliability and veracity of the bioinformatics analysis results.

Human Kidney Tissue Sample Collection

Renal cortex tissues surrounding a cyst were obtained from ADPKD patients undergoing nephrectomy. As a control group, noncancerous renal cortex tissues were acquired from renal cell carcinoma patients undergoing surgical treatment; malignant cell infiltration was excluded by histology [22]. All tissues were devoid of bacterial, viral, or other contamination and stored in liquid nitrogen before further processing. All human kidney tissues were obtained in accordance with ethical standards and with approval of the Changzheng Hospital Ethics Committee (Shanghai, China) in accordance with Helsinki Declaration of 2000. Informed consent was obtained from all patients.

Mouse Kidney Tissue Sample Collection

To generate early-onset ADPKD mouse model, $P k d 1^{f l / f l}$ : tamoxifen-Cre positive mice were given an intraperitoneal injection of tamoxifen ( $10 \mathrm{mg} / \mathrm{kg}$ body weight, formulated in corn oil, T5648, Sigma-Aldrich, St. Louis, MO, USA) on postnatal 10 day (P10) to induce Pkd1 deletion in the PKD group. $P k d 1^{f l / f l}$ : tamoxifen-Cre negative mice were used as the control group. At 4 weeks of age (P28), mice were anesthetized by ether before death cervical dislocation for collection of kidney samples [17]. To generate late-onset ADPKD mouse model, $P k d 1^{f / f f}$ : tamoxifen-Cre positive mice were given an intraperitoneal injection of tamoxifen $(125 \mathrm{mg} / \mathrm{kg}$ body weight, formulated in corn oil) on 2 sequential days (P28 and P29) to induce Pkd1 deletion in the PKD group. On postnatal 90 and 180 days of age (P90 and P180), mice were anesthetized by ether before death cervical dislocation 
Table 1. Primer sets for quantitative real-time PCR

\begin{tabular}{|c|c|c|c|}
\hline Gene term & Species & Primer F $5^{\prime}-3^{\prime}$ & Primer R $5^{\prime}-3^{\prime}$ \\
\hline \multirow[t]{3}{*}{$\mathrm{DCN}$} & Mouse & ATGTGGGTGTCAGCTGGATGCG & TGCAGCCCAGGCAAAAGGGT \\
\hline & Human & AGCTTTGAGGGCTCCTGTGGCA & TTGACAGCGGAAGGGGCACA \\
\hline & Zebrafish & GCTTTTGCTGATCTGAAGAGGGTCT & CTGCGGTCACTTTGGTGATCTTGTT \\
\hline \multirow[t]{2}{*}{ TAGLN } & Mouse & TCACTGCCTAGGCGGCCTTT & TCGGCTCATGCCGTAGGATGGA \\
\hline & Human & ATGCCCCGGATGACTTGGCT & TTGGACTGCACTTCGCGGCT \\
\hline \multirow[t]{2}{*}{ AGXT2 } & Mouse & ACAGCCGTGTGCTGGCGATT & TCGGGTGGCAGTGACCAACA \\
\hline & Human & TGGCTCTTTGATGCTGAAGG & AGAAGACGGTGCTTGTATGC \\
\hline \multirow[t]{2}{*}{ GAPDH } & Mouse & AGAACATCATCCCTGCATCC & ATACCAGGAAATGAGCTTGAC \\
\hline & Human & TAGCCGCATCTTCTTTTGCG & GCGCCCAATACGACCAAATC \\
\hline \multirow[t]{2}{*}{ EHHADH } & Mouse & AGAGGTCATTCCTAGCCGAT & ACATCCTCTGGCTTACTACCTT \\
\hline & Human & GCTTCTTCCACTGATCGTCCTC & CСССАСАAАTCСAAАACAGTTGC \\
\hline \multirow[t]{2}{*}{ HADH } & Mouse & ACCAGACAAGACCGATTTGCT & ACCAAGAGTCGGTTCACGAT \\
\hline & Human & TGGCCTCCATTTCTTCAACCC & TCGTTCATACAGCCTGATTGCTT \\
\hline \multirow[t]{3}{*}{ ALB $\beta$-actin } & Mouse & CTGCGCTGAAGCCAATCCTC & TTTCTTGCAGCCTCCACGAGA \\
\hline & Human & CCAGAATGCGCTATTAGTTCGT & ACTGGCGTTTTCTCATGCAAC \\
\hline & Zebrafish & TTACCACTTCACGCCGACTC & GTCACCTTCACCGTTCCAGT \\
\hline
\end{tabular}

ALB, albumin; HADH, hydroxyacyl-CoA dehydrogenase; EHHADH, enoyl-coenzyme A hydratase/3hydroxyacyl coenzyme A dehydrogenase; AGXT2, alanine-glyoxylate aminotransferase 2; TAGLN, transgelin; DCN, decorin; PCR, polymerase chain reaction.

for collection of kidney samples individually. All kidneys were processed for RNA or protein extraction (flash frozen in liquid nitrogen and stored at $-80^{\circ} \mathrm{C}$ ) and histologic examination (fixed in $4 \%$ paraformaldehyde buffered solution, $\mathrm{pH}$ 7.3-7.4). All mice were raised in specific pathogen-free animal facilities according to a protocol approved by the Animal Care Committee of the Second Military Medical University, Shanghai, China.

\section{Zebrafish Sample Collection}

Wild-type zebrafish (Nüsslein-Volhard Laboratory, Tübingen, Germany) were maintained as previously described [33, 34]. Embryos were obtained from natural mating and were kept in E3 solution at $28.5^{\circ} \mathrm{C}$ [35]. The $p k d_{2}$ morpholino (MO) were injected into embryos at the one cell stage to generate ADPKD zebrafish model. Antisense MO oligos were synthesized by Gene Tools (Philomath, OR, USA). Sequences of the $p k d_{2}$ MO were used as described: $5^{\prime}$-AGGACGAACGCGACTGGAGCTCATC-3'; and negative control MO was used as described: 5'-CCTCTTACCTCAGTTACAATTTATA-3' [35].

Quantitative Real-Time PCR

Total RNA was extracted from human or mouse kidney tissue samples and zebrafish using Trizol reagent (Takara, Shiga, Japan), according to the manufacturer's instructions. The cDNA was synthesized with $2 \mu \mathrm{g}$ of total RNA, cDNA synthesis mix (Biomake), and oligo (dT) primers. The primer sets used for the albumin (ALB), enoyl-coenzyme A hydratase/3-hydroxyacyl coenzyme A dehydrogenase (EHHADH), hydroxyacylCoA dehydrogenase (HADH), decorin (DCN), transgelin (TAGLN), and alanine-glyoxylate aminotransferase 2 (AGXT2) genes are listed in Table 1. GAPDH or $\beta$-ACTIN was used as the endogenous control. Gene expression data were obtained using a real-time PCR assay (Biomake) and a 7900HT real-time PCR system (Applied Biosystems, Foster City, CA, USA). The relative amount of mRNA compared to the internal control was calculated using the $2^{-\Delta C T}$ method, in which $\Delta-C T=-\Delta \mathrm{CT}_{\text {experimental }}-\Delta-\mathrm{CT}_{\text {control }}[36]$. 
Kidney

Blood Pressure

Research

\begin{tabular}{l|l}
\hline Kidney Blood Press Res 2019;44:533-552 \\
\hline DOI: 10.1159/000500458 & $\begin{array}{l}\text { @ 2019 The Author(s). Published by S. Karger AG, Basel } \\
\text { www.karger.com/kbr }\end{array}$ \\
\hline
\end{tabular}

Liu et al.: Bioinformatics Analysis of ADPKD

A

\section{GSE 35831 up-regulated}

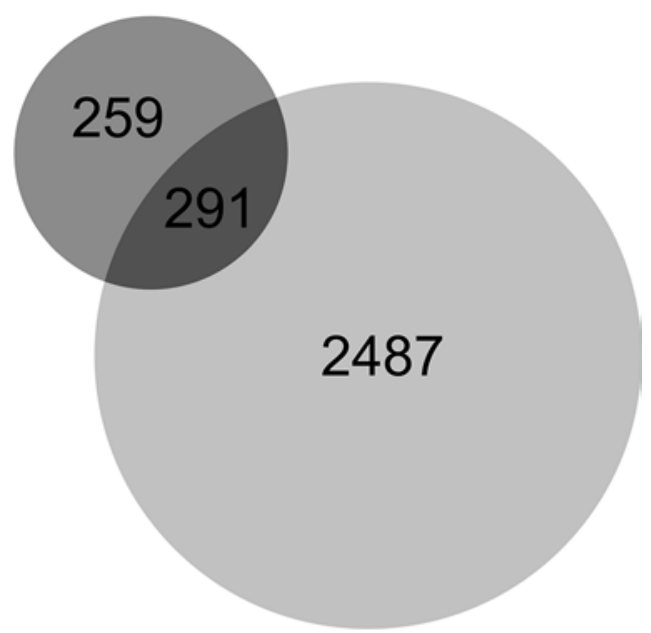

B

GSE 7869 up-regulated

\section{GSE 35831 down-regulated}

Fig. 1. Total of 561 consistently expressed genes identified from GSE7869 and GSE35831, including 291 upregulated genes (A) and 270 downregulated genes (B) in ADPKD kidney tissues compared to control. Different color areas represented different datasets. The cross areas meant the consistently expressed genes.

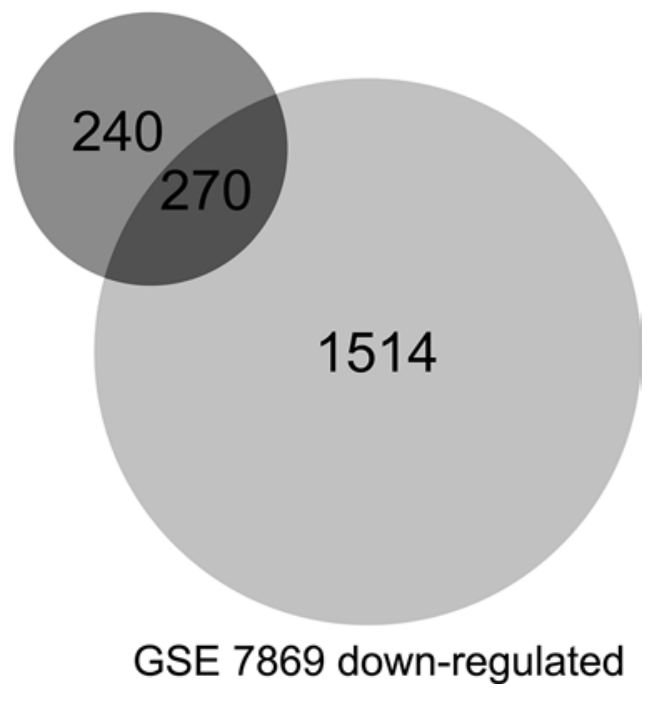

Immunohistochemistry Staining

All kidney tissues were embedded in paraffin and cut into a thickness of $3 \mu \mathrm{m}$ sections. The kidney sections were deparaffinized and rehydrated. Immunohistochemistry (IHC) staining was performed as described previously [37], with the anti-goat DCN (mouse-AF1060, 1:20, human-AF143, 1:15, R\&D, USA).

Statistical Analysis

All data are shown as mean \pm SEM. For data that were normally distributed, comparisons between 2 groups were conducted using the two-tailed $t$ test. Wilcoxon tests were used for skewed continuous data. A $p$ value $<0.05$ was regarded as statistically significant. All analyses were conducted using SPSS 18.0 (SPSS Inc., Chicago, IL, USA). Graphs were constructed using GraphPad Prism software version 5.0 (GraphPad Software, Inc., San Diego, CA, USA). 


\section{Kidney \\ Blood Pressure \\ Research}

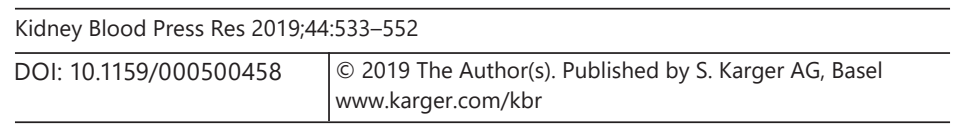

Liu et al.: Bioinformatics Analysis of ADPKD

Table 2. Total of 561 consistently expressed DEGs identified from GSE7869 and GSE35831, according to integrated bioinformatics analysis, including 291 upregulated genes and 270 downregulated genes in ADPKD kidney tissues, compared to normal kidney tissues

\begin{tabular}{|c|c|}
\hline DEGs & Gene name \\
\hline Upregulated & $\begin{array}{l}\text { SAMSN1, OLFML3, CXCL2, FAM129B, SRGN, ATP8B2, NXPE3, BCAT1, ATAD2, LY96, ALDH1L2, RAB31, } \\
\text { NDN, EHD2, TTC39C, TNFAIP8, FGF2, EFEMP1, GUCY1A3, CD86, ENO2, RNF144B, RGS1, FOS, FAM43A, } \\
\text { SBSPON, LAPTM5, PTCH1, TGFB1, LM03, PIK3IP1, ST8SIA4, TIMP1, ABCG1, ATRNL1, RPS9, PLD3, } \\
\text { APOBEC3C, GIMAP2, FLNA, TAGLN, FGF7, MEF2C, SPON1, CLEC2B, RFTN1, TUSC3, HEPH, EGR1, } \\
\text { HDGFRP3, FEZ1, ITGBL1, ANXA1, MDFIC, LHFP, RUNX1T1, BCL6, PDGFD, SPARC, CTSS, DCN, PTPRC, } \\
\text { TIMP2, NCF2, KIAA1551, MYH11, DDIT4, DPYSL3, KDELR3, FCGR2B, FXYD1, C1S, ARL4C, GNG11, } \\
\text { ACTG2, ECM2, GRK5, MLLT3, CARD6, DOCK11, ADH1C, PDLIM1, CYR61, NR2F2, TMEM43, TPM1, } \\
\text { CCDC80, TUBA1A, LRRC17, AMOTL2, ITPRIPL2, LUM, NAP1L1, ADH1B, ID4, NUPR1, DPYSL2, SLPI, } \\
\text { VWF, ENTPD1, PDE5A, FMOD, NCKAP1L, CYBRD1, SSPN, KIAA1462, PDGFRL, EVI2B, PKN1, BTG1, } \\
\text { RASSF2, PTRF, BMP4, ARRB1, NALCN, FCGR2A, AXL, CD44, MYL9, GSTM5, MFGE8, IGF1, SNX33, PERP, } \\
\text { FSTL1, SMOC2, MY01D, KLF4, SLC4A11, FN1, CCL28, CFH, CXCR4, ELN, C1QA, VIM, TGFB3, SYT11, } \\
\text { ADAMTS5, PLK2, CYP1B1, KANK2, MOXD1 }\end{array}$ \\
\hline Downregulated & $\begin{array}{l}\text { PLG, TRPM3, SERPINF2, CTXN3, ZYG11A, PTPN20, CDHR2, CDHR5, HNF4A, PVALB, TFEC, SLC5A11, } \\
\text { TUBAL3, PROC, SLC25A42, TCL6, SLC34A3, KCNAB2, SLC7A13, CRIPAK, ZDHHC23, GJB2, SH3YL1, } \\
\text { DNASE1, FCAMR, PIPOX, SLC22A8, NAT8B, AP0E, HDHD3, AFM, KHK, GLYCTK, UPP2, LEAP2, MSRA, } \\
\text { SLC39A5, PCK2, SLC5A2, ECHS1, CTSA, SNX30, ANKS4B, SLC22A12, CYP2B6, PRODH2, AGMAT, } \\
\text { PDZK1IP1, CCDC88C, CLNK, HA02, NQ02, AGT, ACSF2, SLC22A5, HNF4G, DMRT2, A1CF, ABCC2, } \\
\text { CYP8B1, FARP1, ECHDC2, SLC23A1, SLC13A3, ANK2, MT1F, SLC2A9, ANGPTL3, TRPM6, AHCY, MIOX, } \\
\text { HAVCR2, GCH1, SLC12A6, ALDOB, LGMN, SLC22A11, AC01, MAPT, TTC38, CYP4F2, TMEM174, TMED6, } \\
\text { CDH9, SLC9A3R1, ACOX2, DI01, TMEM207, IGSF11, SLC16A1, SLC25A5, PPARA, SLC2A2, GLB1L, } \\
\text { SLC7A9, AKR1A1, TPCN2, FTCD, HIBCH, MY0M3, CYP4F3, VIL1, PDSS1, CYP27B1, SLC22A6, SLC13A2, } \\
\text { SLC5A12,SYT13, STRIP2, CYP17A1, SLC6A19, SLC10A2, SLC22A7, AGXT2, SLC5A9, SLC28A1, } \\
\text { PLA2G12B, SLC23A3, SLC16A10, SLC22A13, SLC1A1, SLC7A7 }\end{array}$ \\
\hline
\end{tabular}

DEGs, differentially expressed genes; ADPKD autosomal dominant polycystic kidney disease.

\section{Results}

\section{Identification of DEGs in ADPKD}

In the comparison of gene expression between ADPKD tissues and normal kidney tissues, 6,422 and 1,152 DEGs extracted from the expression profile datasets GSE7869 and GSE35831, respectively. By utilized Funrich_V3, 561 DEGs were identified in ADPKD kidney tissues, which were consistently expressed in both GSE7869 and GSE35831, including 291 upregulated genes and 270 downregulated genes, compared to normal kidney tissues (Fig. 1; Table 2).

\section{GO Analysis of DEGs}

The functions and pathways of candidate DEGs were obtained by using multiple online websites, including DAVID, KEGG pathway, and GO, and the cutoff criterion was $p<0.05$ [26, $29,30]$. GO analysis was performed with DAVID. The DEGs were classified into 3 functional groups [29,30]: molecular function, biological process, and cellular component (Fig. 2, 3), and the DEGs that showed significant enrichment are listed in Table 3.

In the biological process group, the upregulated genes (Fig. 2A, 3A) were mainly involved in extracellular matrix (ECM) organization, cell adhesion, ECM disassembly, muscle contraction, and wound healing. In the molecular function group, the upregulated genes were mainly linked to protein binding, heparin binding, collagen binding, and calcium ion binding. In the cellular 
Kidney

Blood Pressure

Research
Kidney Blood Press Res 2019;44:533-552

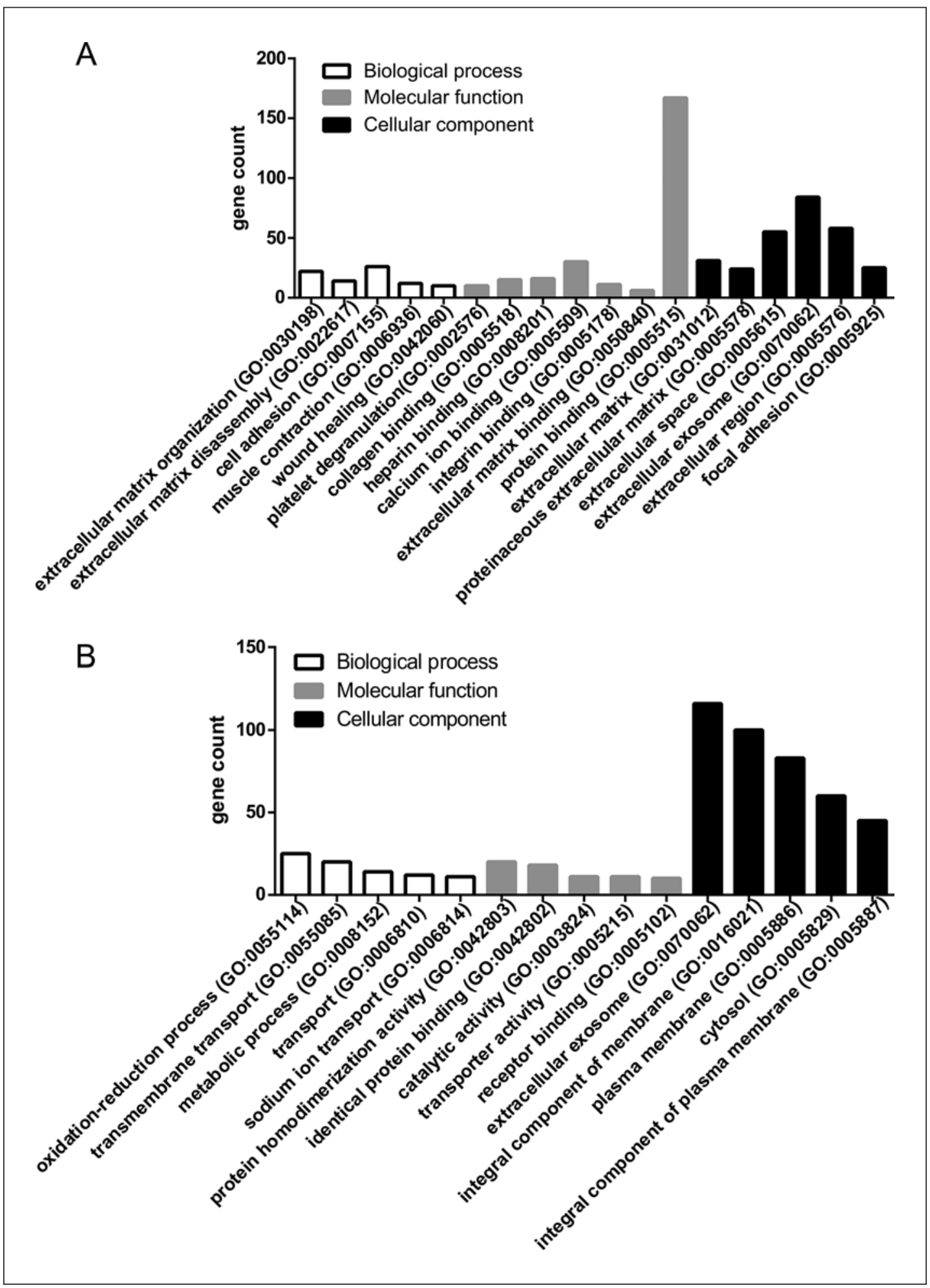

Fig. 2. GO analysis of consistently expressed DEGs in ADPKD. Upregulated DEGs (A) and downregulated DEGs (B) were individually classified into 3 groups: biological process (white), molecular function (gray), and cellular component (black). GO, gene ontology. 
A

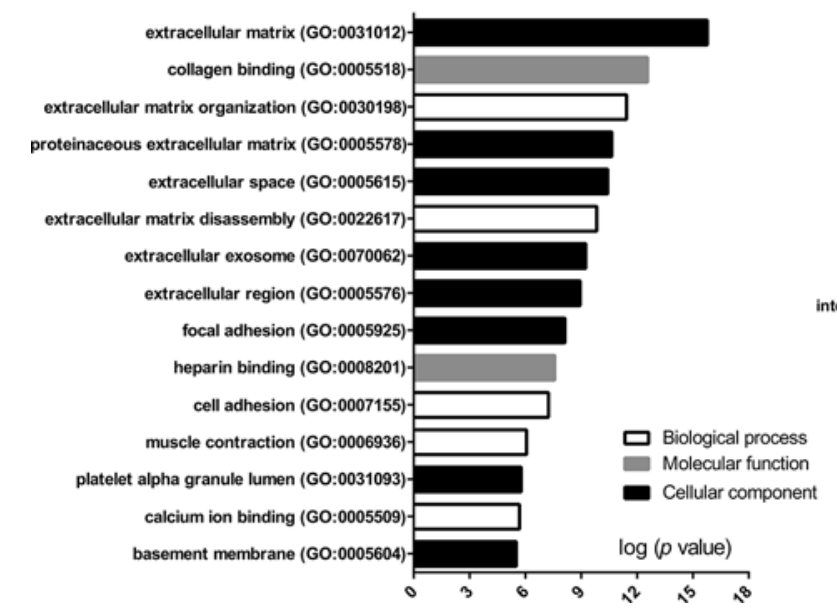

B

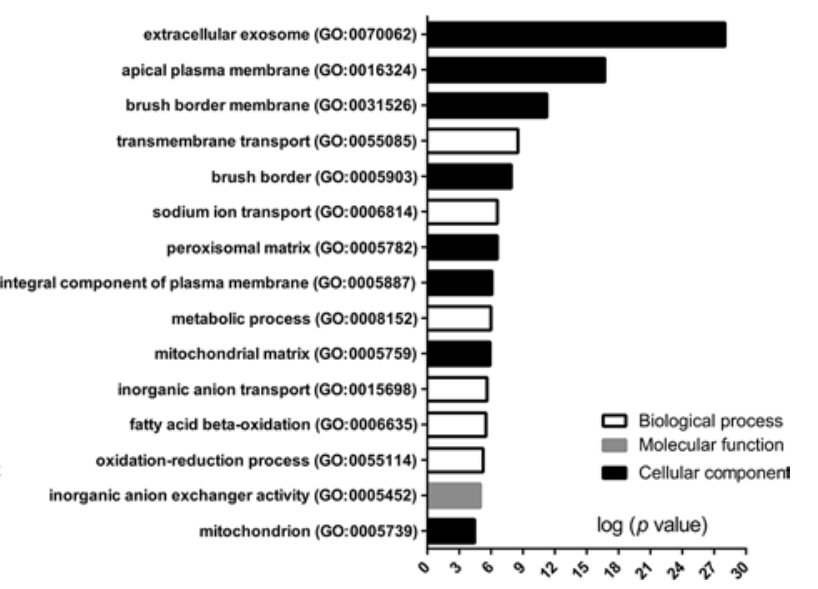

Fig. 3. Significant Enriched Go Terms of consistently expressed DEGs in ADPKD based on their functions, including upregulated DEGs (A) and downregulated DEGs (B). GO, gene ontology.

Table 3. The significant enriched analysis of DEGs in ADPKD

\begin{tabular}{llrl}
\hline Term & Description & Count & $p$ value \\
\hline Up-regulated & & & \\
GO:0031012 & Extracellular matrix & 31 & $1.6658 \mathrm{E}-16$ \\
GO:0005518 & Collagen binding & 15 & $2.74844 \mathrm{E}-13$ \\
GO:0030198 & Extracellular matrix organization & 22 & $3.69419 \mathrm{E}-12$ \\
GO:0005578 & Proteinaceous extracellular matrix & 24 & $2.26852 \mathrm{E}-11$ \\
GO:0005615 & Extracellular space & 55 & $3.72548 \mathrm{E}-11$ \\
GO:0022617 & Extracellular matrix disassembly & 14 & $1.46593 \mathrm{E}-10$ \\
GO:0070062 & Extracellular exosome & 84 & $5.73833 \mathrm{E}-10$ \\
GO:0005576 & Extracellular region & 58 & $1.13345 \mathrm{E}-09$ \\
GO:0005925 & Focal adhesion & 25 & $7.56565 \mathrm{E}-09$ \\
GO:0008201 & Heparin binding & 16 & $2.6642 \mathrm{E}-08$ \\
\hline Down-regulated & & & \\
GO:0070062 & Extracellular exosome & 116 & $9.39062 \mathrm{E}-29$ \\
GO:0016324 & Apical plasma membrane & 31 & $1.99271 \mathrm{E}-17$ \\
GO:0031526 & Brush border membrane & 13 & $5.59467 \mathrm{E}-12$ \\
GO:0055085 & Transmembrane transport & 20 & $2.83982 \mathrm{E}-09$ \\
GO:0005903 & Brush border & 11 & $1.22805 \mathrm{E}-08$ \\
GO:0006814 & Sodium ion transport & 11 & $2.55632 \mathrm{E}-07$ \\
GO:0005782 & Peroxisomal matrix & 9 & $2.59143 \mathrm{E}-07$ \\
GO:0005887 & Integral component of plasma membrane & 15 & $8.17944 \mathrm{E}-07$ \\
GO:0008152 & METABOLIC process & 19 & $1.03062 \mathrm{E}-06$ \\
GO:0005759 & Mitochondrial matrix & $1.23057 \mathrm{E}-06$ \\
\hline
\end{tabular}

DEGs, differentially expressed genes; ADPKD autosomal dominant polycystic kidney disease. 


\section{Kidney \\ Blood Pressure \\ Research}

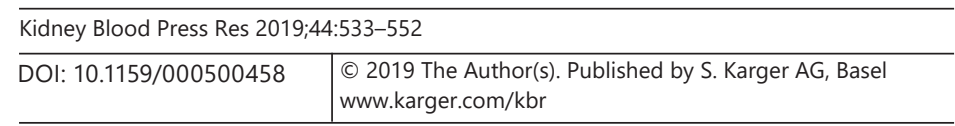

Liu et al.: Bioinformatics Analysis of ADPKD

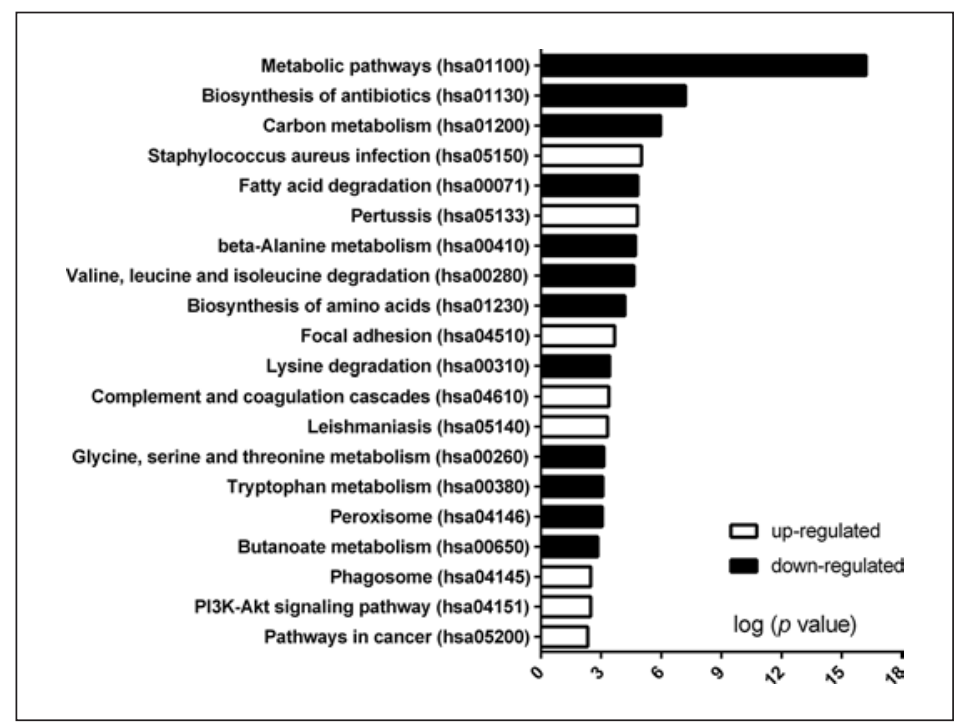

Fig. 4. Significant enriched pathway terms of DEGs (upregulated, white; downregulated, black).

component group, the upregulated genes were mainly related to the extracellular exosome, extracellular region, proteinaceous ECM, extracellular space, and focal adhesion.

In the biological process group, the downregulated genes (Fig. 2B, 3B) were mainly involved in transmembrane transport, oxidation-reduction process, metabolic process, sodium ion transport, and fatty acid $\beta$-oxidation. In the molecular function group, the downregulated genes were mainly associated with protein homodimerization activity, identical protein binding, catalytic activity, transporter activity, oxidoreductase activity, hydrolase activity, and receptor binding. In the cellular component group, the downregulated genes were mainly related to the extracellular exosome, integral component of the membrane, plasma membrane, cytosol, integral component of the plasma membrane, mitochondrion, apical plasma membrane, and endoplasmic reticulum membrane.

\section{Signaling Pathway Enrichment Analysis}

The functional and signaling pathways enriched in association with the DEGs were investigated through the KEGG pathway and GO online tools (Fig. 4; Table 4). The upregulated DEGs were mainly related to Staphylococcus aureus infection, pertussis, phagosome, pathways in cancer, PI3K-Akt signaling pathway, focal adhesion, proteoglycans in cancer, complement, and coagulation cascades. The downregulated DEGs were mainly involved in metabolic pathways; biosynthesis of antibiotics; carbon metabolism; fatty acid degradation; $\beta$-alanine metabolism; lysine, valine, and (iso) leucine degradation; glycine, serine, and threonine metabolism; and biosynthesis of amino acids.

\section{PPI Network and Modular Analysis of Key GENES and Pathways}

The String online database [25] and Cytoscape software [38] were used for PPI and modular analysis. A total of 561 consistently expressed DEGs (291 upregulated and 270 downregulated genes) were individually filtered into the PPI network, and 421 nodes and 1,776 edges (Fig. 5A) were obtained. From the 421 nodes, 34 central node genes were acquired, and the 10 most significant node genes were $A L B, J U N$, insulin-like growth factor 1, fibroblast growth factor 2, alpha smooth muscle actin 2, plasminogen, EHHADH, vimentin, and DCN. According to the degree of importance, 2 significant modules were selected from the PPI network for further analysis using Cytotype MCODE. Pathway enrichment analysis showed that 23 nodes and 140 edges (Fig. 5B; 
Kidney
Blood Pressure

Research
Kidney Blood Press Res 2019;44:533-552

DOI: $10.1159 / 000500458$

c 2019 The Author(s). Published by S. Karger AG, Base

www.karger.com/kbr

Liu et al.: Bioinformatics Analysis of ADPKD

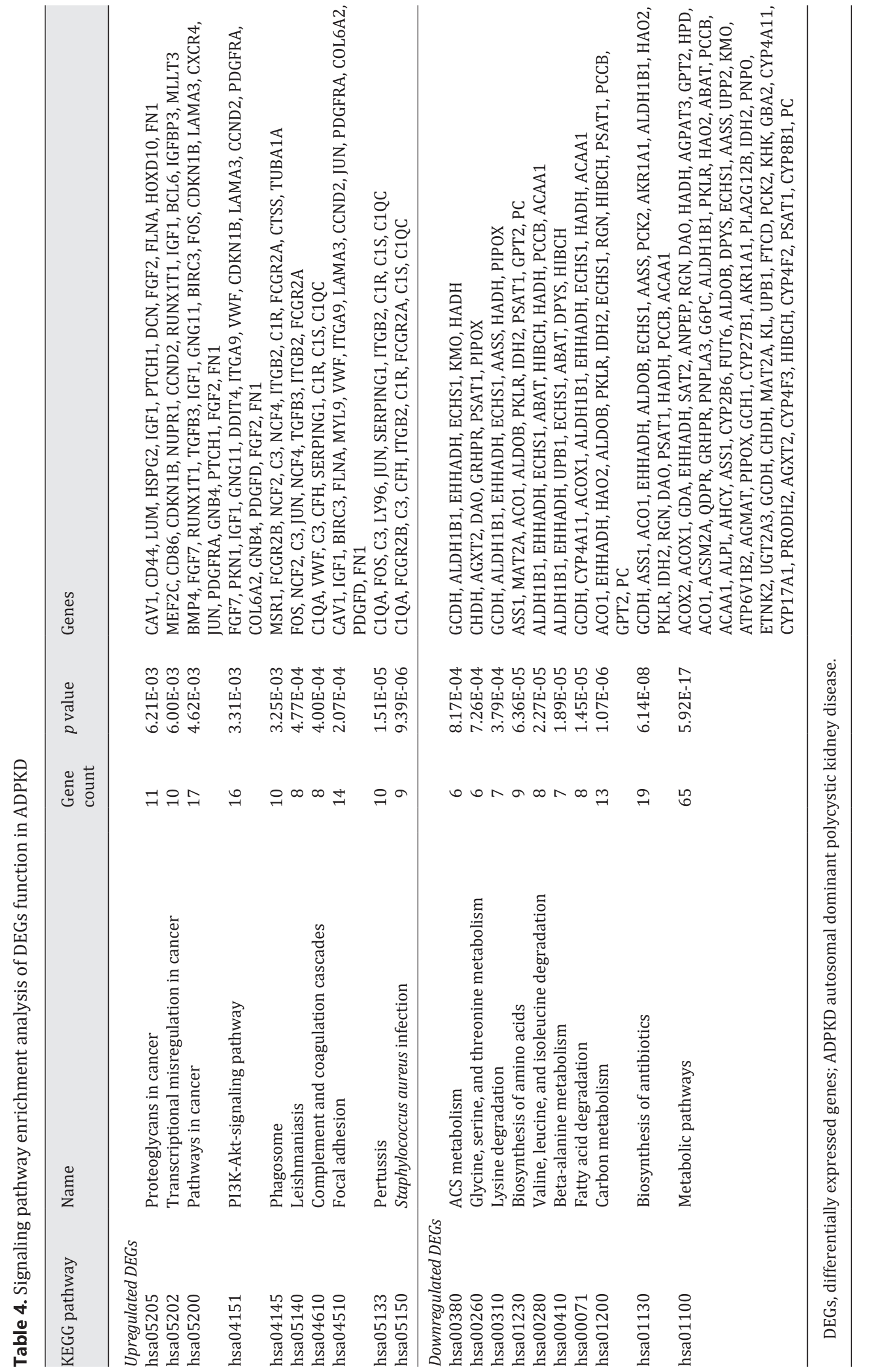




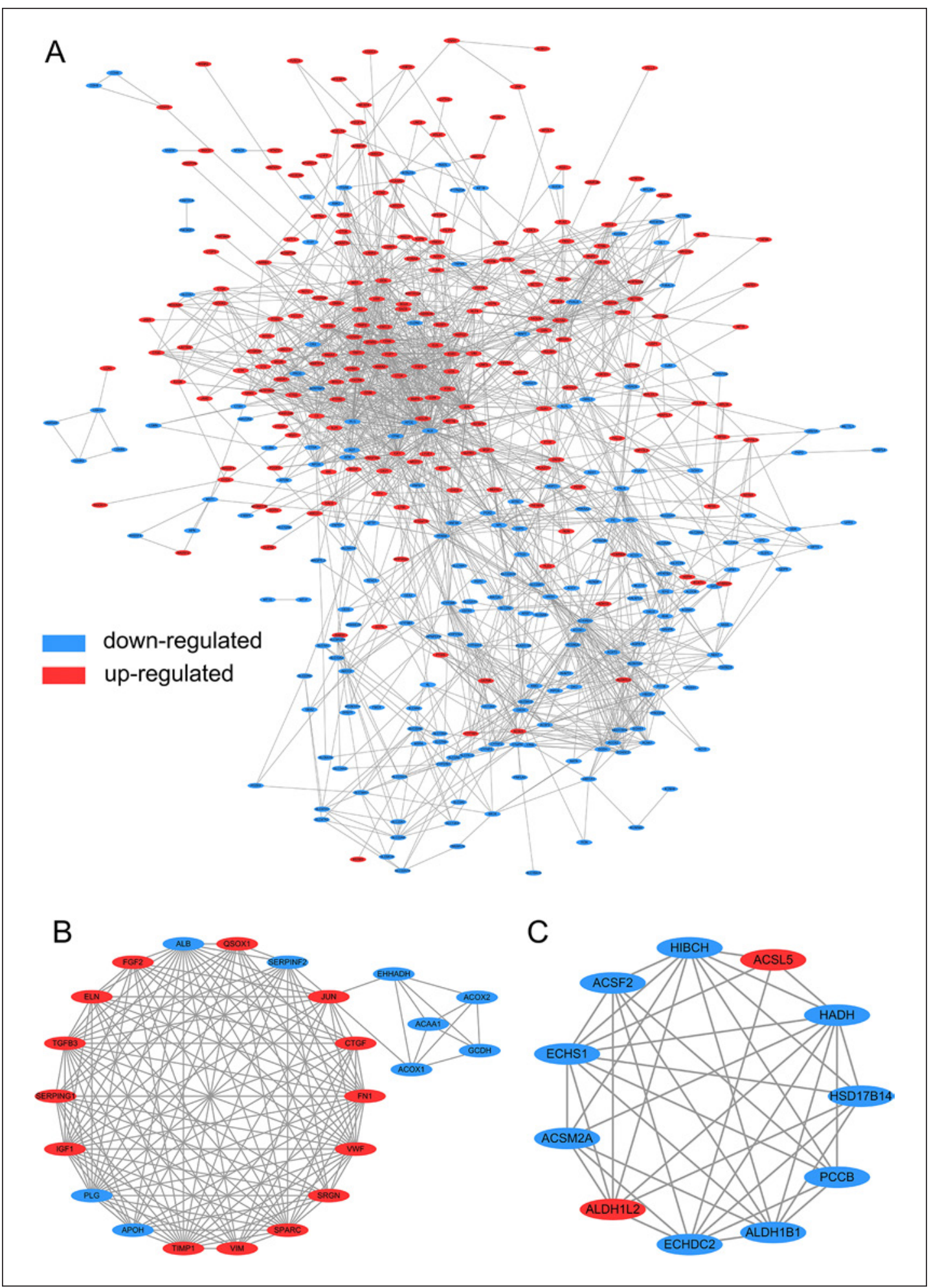

Fig. 5. PPI network complex and modular analysis of different express genes. A Total of 561 DEGs (291 upregulated genes in red and 270 downregulated genes in blue) filtered into the DEGs PPI network complex by using the STRING online database, includes of 421 nodes and 1,776 edges. B Module 1 includes of 23 nodes and 140 edges. C Module 2 includes of 11 nodes and 39 edges. 
Table 5. Pathway enrichment analysis of module 1 gene function

\begin{tabular}{|c|c|c|c|}
\hline Term & Description & Count & $p$ value \\
\hline GO:0005515 & Protein binding & 19 & 0.0000759 \\
\hline GO:0005576 & Extracellular region & 16 & $1.13 \mathrm{E}-14$ \\
\hline GO:0005615 & Extracellular space & 14 & $9.92 \mathrm{E}-13$ \\
\hline GO:0002576 & Platelet degranulation & 13 & $7.52 \mathrm{E}-27$ \\
\hline GO:0031093 & Platelet alpha granule lumen & 12 & $3.02 \mathrm{E}-28$ \\
\hline GO:0070062 & Extracellular exosome & 10 & 0.000178225 \\
\hline GO:0005886 & Plasma membrane & 8 & 0.038480439 \\
\hline GO:0042802 & Identical protein binding & 7 & 0.000000342 \\
\hline GO:0007568 & Aging & 6 & $8.75 \mathrm{E}-10$ \\
\hline GO:0042060 & Wound healing & 5 & $4.50 \mathrm{E}-10$ \\
\hline GO:0005777 & Peroxisome & 5 & $2.41 \mathrm{E}-09$ \\
\hline GO:0072562 & Blood microparticle & 5 & $1.39 \mathrm{E}-08$ \\
\hline G0:0008083 & Growth factor activity & 5 & $3.22 \mathrm{E}-08$ \\
\hline GO:0030198 & Extracellular matrix organization & 5 & 8.95E-08 \\
\hline G0:0008284 & Positive regulation of cell proliferation & 5 & 0.0000135 \\
\hline GO:0043231 & Intracellular membrane-bounded organelle & 5 & 0.0000365 \\
\hline GO:0009986 & Cell surface & 5 & 0.0000393 \\
\hline GO:0045944 & Positive regulation of transcription from RNA polymerase II promoter & 5 & 0.000788458 \\
\hline GO:0005739 & Mitochondrion & 5 & 0.003013631 \\
\hline hsa05200 & Pathways in cancer & 5 & 0.000322875 \\
\hline hsa00071 & Fatty acid degradation & 4 & $6.11 \mathrm{E}-08$ \\
\hline hsa03320 & PPAR signaling pathway & 4 & 0.000000608 \\
\hline hsa04610 & Complement and coagulation cascades & 4 & 0.0000012 \\
\hline hsa04146 & Peroxisome & 4 & 0.00000154 \\
\hline hsa04510 & Focal adhesion & 4 & 0.000117309 \\
\hline hsa04151 & PI3K-Akt signaling pathway & 4 & 0.001318973 \\
\hline
\end{tabular}

GO, gene ontology.

Table 6. Pathway enrichment analysis of module 2 gene function

\begin{tabular}{llll}
\hline Term & Description & Count & $p$ value \\
\hline G0:0005739 & Mitochondrion & 8 & $1.06 \mathrm{E}-09$ \\
hsa00280 & Valine, leucine, and isoleucine degradation & 5 & $2.02 \mathrm{E}-12$ \\
hsa00071 & Fatty acid degradation & 4 & $4.18 \mathrm{E}-10$ \\
G0:0005524 & ATP binding & 4 & 0.00092 \\
hsa00380 & Tryptophan metabolism & 3 & $6.17 \mathrm{E}-08$ \\
hsa00640 & Propanoate metabolism & 3 & $2.44 \mathrm{E}-08$ \\
hsa00310 & Lysine degradation & 3 & $1.82 \mathrm{E}-07$ \\
hsa01212 & Fatty acid metabolism & 3 & $1.31 \mathrm{E}-07$ \\
hsa01200 & Carbon metabolism & 3 & $4.20 \mathrm{E}-06$ \\
hsa00650 & Butanoate metabolism & 3 & $1.61 \mathrm{E}-08$ \\
hsa00410 & Beta-alanine metabolism & 3 & $2.13 \mathrm{E}-08$ \\
G0:0006631 & Fatty acid metabolic process & 3 & $1.88 \mathrm{E}-08$ \\
\hline
\end{tabular}

GO, gene ontology. 
Kidney

Blood Pressure

Research
Kidney Blood Press Res 2019;44:533-552

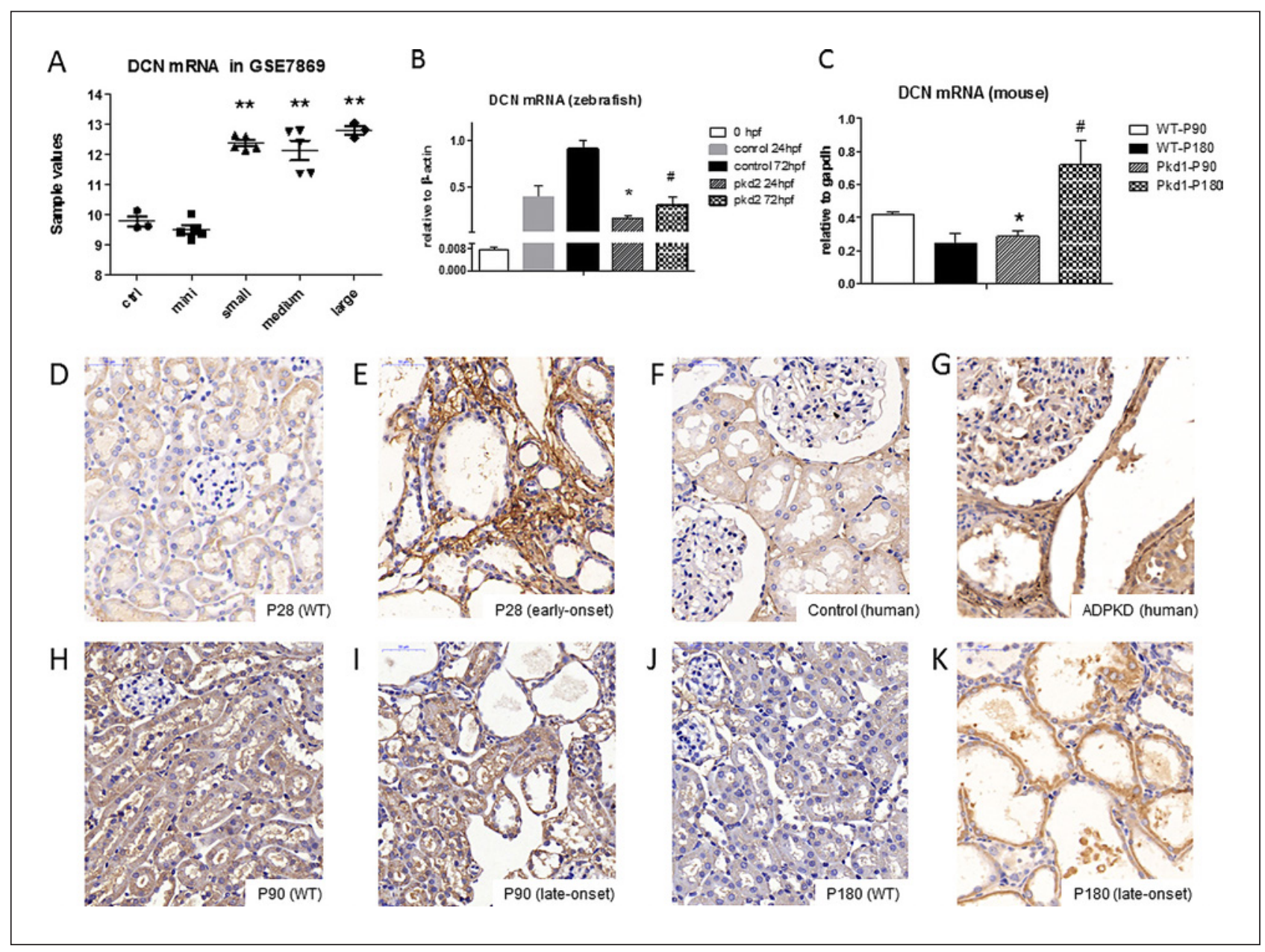

Fig. 6. DCN expression in ADPKD renal tissues. A DCN mRNA expression values (sample values for ID = 209335 at) in GSE7869. DCN mRNA expression values were upregulated in small, medium and large cysts groups compared to control and minimally groups. Data are expressed as mean $\pm \mathrm{SEM}$; ${ }^{* *} p<0.01$ versus or Control or minimally groups. B DCN mRNA expression in ADPKD zebrafish model. DCN mRNA expressions were downregulated in zebrafish model of ADPKD (pkd2 morphants) groups compared to control groups after $24 \mathrm{hpf}$ (hour post fertilization) and 72 hpf. Data are expressed as mean \pm SEM; ${ }^{*} p<0.05$ versus Control 24 hpf; ${ }^{*} p<0.05$ versus control $72 \mathrm{hpf}$. C Renal DCN mRNA expressions in late-onset ADPKD mouse model. On P90, renal. DCN mRNA expressions were downregulated in Pkd1 mouse group compared to WT group. On P180, renal DCN mRNA expressions were upregulated in Pkd1 mouse group compared to WT group. Data are expressed as mean $\pm \mathrm{SEM}$; ${ }^{*} p<0.05$ versus WT; ${ }^{*} p<0.05$ versus WT. D-K Renal DCN protein expression in ADPKD. In ADPKD early-onset mouse model, a marked increased expression of DCN protein was observed in renal interstitial tissue compared to WT mouse on postnatal 28 day (P28; D, E). In ADPKD patient renal tissue, significant increased expression of DCN protein was observed in renal interstitial tissue and CLECs compared to control renal tissue $(\mathbf{F}, \mathbf{G})$. There was no difference on DCN protein expression in renal tissue between ADPKD late-onset mouse and WT mouse on P90 (H, I). In ADPKD late-onset mouse model, increased expression of DCN protein was observed in CLECs and renal interstitial tissue compared to WT mouse on P180 (J, K; ×400). DCN, decorin.

Table 5) constituted module 1 and were associated with protein binding, extracellular region, extracellular space, platelet degranulation, extracellular exosome, plasma membrane, and aging. Module 2 consisted of 11 nodes and 39 edges associated with mitochondrion, degradation of valine and (iso) leucine, fatty acid degradation, ATP binding, tryptophan metabolism, propanoate metabolism, lysine degradation, fatty acid, and carbon metabolism (Fig. 5C; Table 6). 


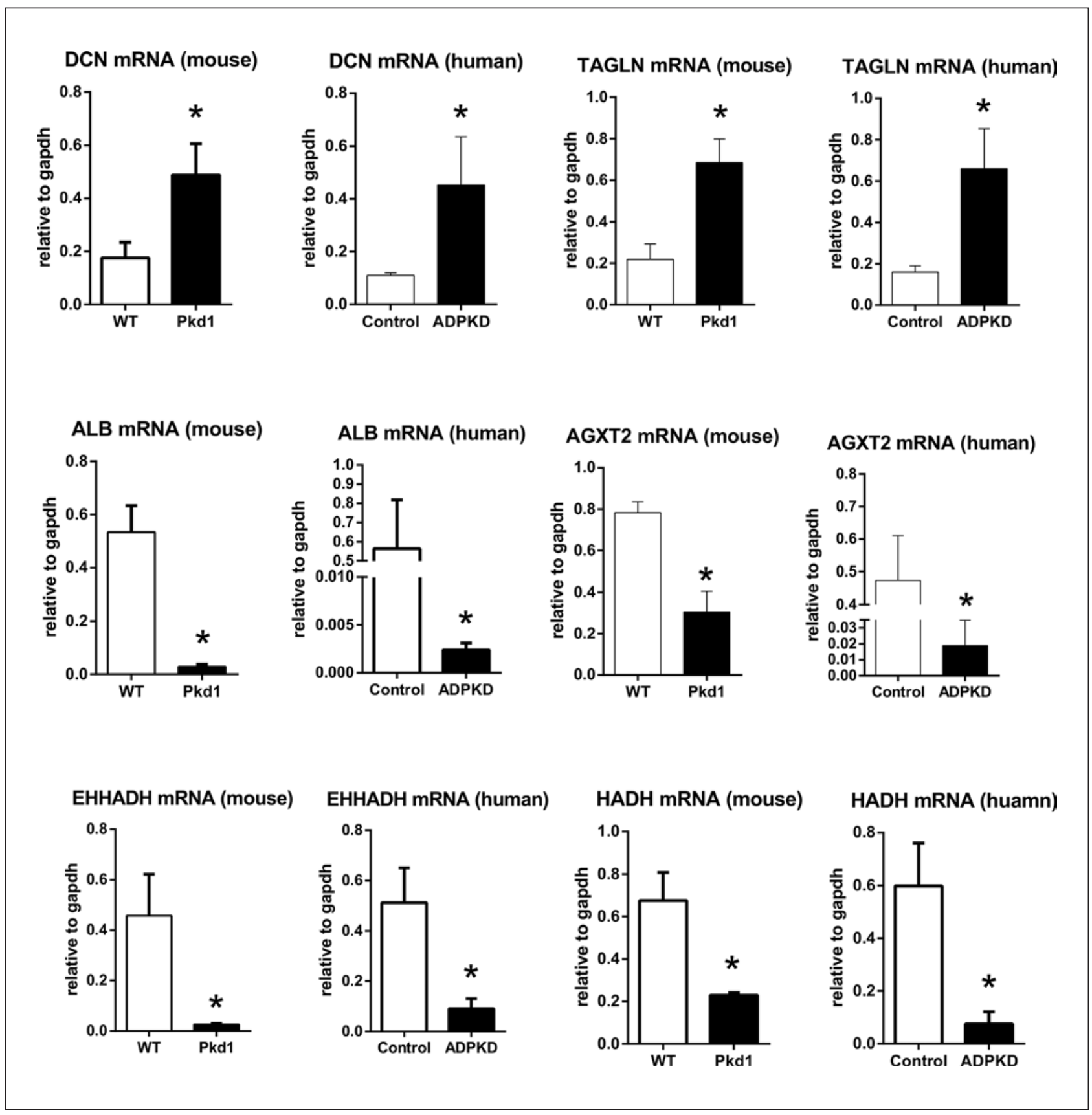

Fig. 7. Quantitative real-time PCR of renal tissue in Pkd1 mouse model and ADPKD human compared to WT and control. Data are expressed as mean \pm SEM; * $p<0.05$ vs. WT or Control. DCN, decorin; TAGLN, transgelin; ALB, albumin; AGXT2, alanine-glyoxylate aminotransferase 2; EHHADH, enoyl-coenzyme A hydratase/3hydroxyacyl coenzyme A dehydrogenase; HADH, hydroxyacyl-CoA dehydrogenase.

DCN mRNA expression values (sample values for ID = 209335_at) were reanalyzed in GSE7869 according to different developmental stage of cysts formation, including control, minimally cystic tissue, small, medium, and large cysts [21]. DCN mRNA expression values were upregulated in small, medium, and large cysts groups compared to control and minimally groups. No difference was observed between control and minimally cystic tissue (Fig. 6A). 


\section{Kidney \\ Blood Pressure \\ Research}

\begin{tabular}{l|l}
\hline Kidney Blood Press Res 2019:44:533-552 \\
\hline DOI: 10.1159/000500458 & $\begin{array}{l}\text { @ 2019 The Author(s). Published by S. Karger AG, Basel } \\
\text { www.karger.com/kbr }\end{array}$ \\
\hline
\end{tabular}

Liu et al.: Bioinformatics Analysis of ADPKD

\section{Validation of Selected DEGs}

Quantitative Real-Time PCR

Using qPCR in kidney samples affected by PKD and normal kidney samples from a mouse model (early-onset ADPKD) and humans, we confirmed the differential expression of DEGs identified via our bioinformatics analysis, including: ALB, EHHADH, HADH, DCN, TAGLN, and AGXT2 (Fig. 7). The expression levels of $D C N$ and TAGLN were significantly higher in PKD kidney samples from both humans and mice compared to levels in control samples. The expression levels of $A L B, E H H A D H, H A D H$, and $A G X T 2$ were significantly lower in PKD kidney samples from both humans and mice compared to levels in control samples.

ADPKD zebrafish model was used to detect DCN mRNA expression in different stages of cyst formation. DCN mRNA expressions were upregulated more than 2 folds in control groups compared to zebrafish model of ADPKD ( $p k d_{2}$ morphants) groups at 24 and $72 \mathrm{hpf}$ (hour post fertilization; Fig. 6B).

Kidney DCN mRNA expressions were detected in late-onset ADPKD mouse model. DCN mRNA of mice kidneys was quantified by qPCR on P90 and P180 individually. On P180, DCN mRNA expressions were upregulated more than 2 folds in $P k d_{1}$ mouse group compared to wide type group (Fig. 6C). On P90, DCN mRNA expressions were downregulated in $P k d_{1}$ mouse group compared to wide-type group, but the change is no more than 2 folds.

\section{IHC Staining}

Renal DCN expression in ADPKD renal tissues was performed in mouse models and human with IHC. In ADPKD early-onset mouse model, a marked increased expression of DCN protein was observed in renal interstitial tissue compared to WT mouse on age of P28 (Fig. 6D, E). In ADPKD late-onset mouse model, increased expression of DCN protein was observed in cyst line epithelial cells and renal interstitial tissue compared to WT mouse on age of P180 (Fig. 6J, K). There was no difference on DCN protein expression in renal tissue between ADPKD late-onset mouse and WT mouse on P90 (Fig. 6H, I). In ADPKD patient renal tissue, significant increased expression of DCN protein was observed in renal interstitial tissue and cyst line epithelial cells compared to control renal tissue (Fig. 6F, G).

\section{Discussion}

Our reanalysis of 2 gene profiling datasets from ADPKD and normal human kidney tissues revealed 561 consistent DEGs (291 upegulated genes and 270 downregulated genes) in ADPKD. Most of upregulated DEGs indicated enrichment of pathways involved in cancer, PI3K-Akt signaling pathway, focal adhesion, complement, and coagulation cascades. Most of downregulated DEGs were associated with enrichment of metabolic pathways, including those responsible for carbon, fatty acid, and amino acid metabolism. The generated PPI network revealed 421 nodes (DEGs), and of these, the 10 most significantly DEGs were validated by qPCR analysis in both a Pkd1 mouse model and ADPKD patient samples.

More specifically, the upregulated DEGs were mainly involved in pathways of ECM organization, ECM disassembly, cell adhesion, collage and integrin binding, protein binding, ECM, and focal adhesion. These findings are consistent with observations in cystic kidneys. For example, Drummond's [39] review of the characteristics of fibrosis observed in cystic kidney diseases provided evidence of altered interactions between epithelial cells and stromal fibroblasts, followed by accumulation of ECM (collagen, specific laminins, and other proteins) and enhanced expression of matrix metalloproteases and transforming growth factor $\beta$. Okada et al. [40] investigated the phenotype of collagen-producing cells in the cystic kidneys of DBA/2pcy mice and characterized the spectrum of interstitial cells associated with renal fibro- 


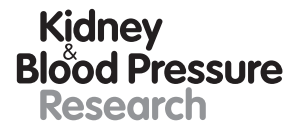

Kidney
Blood Pressure
Research \begin{tabular}{l|l}
\hline Kidney Blood Press Res 2019;44:533-552 \\
\hline DOI: 10.1159/000500458 & $\begin{array}{l}\text { @ 2019 The Author(s). Published by S. Karger AG, Basel } \\
\text { www.karger.com/kbr }\end{array}$ \\
\hline
\end{tabular}

Liu et al.: Bioinformatics Analysis of ADPKD

genesis. Furthermore, Lee et al. [41] provided genetic evidence that a functional integrin- $\beta 1$ is required for the early events leading to renal cystogenesis in ADPKD and suggested that the integrin signaling pathway may represent an effective therapeutic target for slowing disease progression.

$D C N$ was among the 10 most significantly DEGs and was significantly upregulated in human ADPKD kidney samples. Our experiments validated that DCN was upregulated in human ADPKD kidney samples and in late stage (P180) of late-onset ADPKD mouse model. The protein product of $D C N$ is a member of the small leucine-rich proteoglycan family of proteins [42]. Diseases associated with DCN protein include corneal dystrophy, congenital stromal, and stromal dystrophy [43]. Previous research showed that DCN suppresses tumorigenic growth, angiogenesis, and prevents metastatic lesions in a variety of in vitro and in vivo tumor models and thus represents a promising therapeutic candidate for combatting solid malignancies [44]. In the normal kidney, DCN presents mainly in the renal interstitium [45]. DCN accumulated in tubular interstitial in several nephropathy appeared to play a crucial role in renal inflammation and fibrogenesis, predicting progression of chronic kidney disease [42]. Soluble DCN is regarded as a signaling molecule and an endogenous ligand of TLR2/ TLR4 in renal inflammation $[46,47]$ and appears to be a potent antifibrosis molecule, acting against renal fibrogenesis directly through receptor-mediated signaling and indirectly in transforming GF $\beta$-dependent and - independent pathways [42]. In unilateral ureteral obstruction mouse, the absence of DCN aggravated renal fibrosis. According to our results, we can make a conclusion that DCN accumulation in ADPKD renal interstitium is associated with the development of cysts in ADPKD. Whether DCN takes part in the fibrosis progression or acts against fibrogenesis in ADPKD is not known, and further experiments are necessary. However, DCN was shown decreased in the zebrafish Pkd2-MO model and in the early-stage (P90) of late-onset ADPKD mouse model. DCN mRNA expressions were downregulated significantly in the zebrafish Pkd2-MO model at different stages of cyst formation. We can speculate that the downregulated DCN is associated with the formation of cysts in ADPKD and that DCN might be a retardant during cysts formation. The cross-breeding between DCN knockout mice and $P k d 1$ conditional knockout mice would be necessary to confirm the unique role of DCN in cysts formation and development in ADPKD.

The downregulated DEGs identified in the present study mostly indicated enrichment of metabolic pathways, such as those for carbon metabolism, fatty acid degradation, and amino acid metabolism. In PPI network, module 2 consisted of 11 nodes, mainly associated with mitochondrion and ATP binding. This finding supports the hypothesis that ADPKD pathogenesis could be causally linked to altered cellular metabolism [12]. A study by Menezes et al. [17] demonstrated that metabolic pathways are key elements in postnatal kidney maturation and early steps of cyst formation in a Pkd1 mouse model of ADPKD. Moreover, a mutation in the PKD1 gene was shown to cause enhanced glycolysis in cells, in a murine model of PKD as well as and in human ADPKD kidneys [48]. Another study also revealed defective glucose metabolism in ADPKD and showed that treatment with 2-deoxy-D-glucose could ameliorate the progression of PKD [49]. These findings led to a new therapeutic approach of targeting the glycolytic pathway for treatment of PKD. At the same time, impaired fatty acid oxidation was discovered in an orthologous mouse model of ADPKD [19]. This was supported by a clinical study that obtained evidence of perturbation in fatty acid metabolism early in ADPKD patients, even in those with preserved kidney function [50]. From these results, the authors concluded that the LOX (5- and 12/15-lipoxygenase) pathways may be potential therapeutic targets for slowing the progression of ADPKD.

Some of the downregulated DEGs and corresponding pathways identified in the present study were associated with amino acid metabolism, such as pathways in amino acid (transmembrane) transport, biosynthesis of amino acids, beta-alanine metabolism, and lysine, 


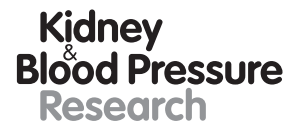

Kidney
Blood Pressure

Research \begin{tabular}{l|l}
\hline Kidney Blood Press Res 2019;44:533-552 \\
\hline DOI: 10.1159/000500458 & $\begin{array}{l}\text { @ 2019 The Author(s). Published by S. Karger AG, Basel } \\
\text { www.karger.com/kbr }\end{array}$ \\
\hline
\end{tabular}

Liu et al.: Bioinformatics Analysis of ADPKD

valine, leucine, and isoleucine degradation. Branched-chain amino acids (BCAAs), including leucine, play crucial roles in the activation of the mammalian target of rapamycin pathway [51], and when Yamamoto et al. [52] administered BCAAs, including leucine, dissolved in the drinking water to a Pkd1 mouse model, they found that BCAAs accelerated ADPKD progression via the mammalian target of rapamycin and MAPK/ERK pathways. Thus, BCAAs may be harmful to patients with ADPKD. Moreover, amino acid metabolism is tightly linked to ADPKD progression. In the present study, we validated the downregulated expression of the EHHADH and $H A D H$ genes in PKD kidney samples compared to control kidney samples. Both of these genes are associated with metabolic pathways, including those for carbon metabolism, fatty acid degradation, and amino acid degradation. Interestingly, mistargeting of peroxisomal EHHADH was found to disrupt mitochondrial metabolism and leads to renal Fanconi's syndrome [53].

Raptis et al. [54] summarized the role of the endothelium in ADPKD. Nitric oxide (NO) bioavailability and NO-dependent vasodilation are affected in patients with ADPKD. Asymmetric dimethylarginine (ADMA) is an endogenous inhibitor of $\mathrm{NO}$ and a putative biochemical marker of atherosclerosis [55]. ADPKD patients with an enhanced glomerular filtration rate $>60 \mathrm{~mL} / \mathrm{min} / 1.73 \mathrm{~m}^{2}$ had higher serum ADMA levels, as well as higher levels of several other endothelial dysfunction markers, than healthy controls [56]. Several studies have noted a possible harmful role of ADMA in ADPKD [57,58]. AGXT2 can metabolize ADMA, and Rodionov et al. [59] found that AGXT2 may play a major role in the maintenance of ADMA homeostasis in the setting of local or systemic elevation of ADMA in mice after bilateral nephrectomy. Our results showed that $A G X T 2$ gene expression was lower by $>20$-fold in ADPKD human kidney samples compared with control tissues and lower by 2 -fold in PKD mice kidney samples compared with wild-type kidney tissues. Thus, we can hypothesize that the increased serum ADMA level in ADPKD patients may correspond with a decline in AGXT2 expression in the affected kidneys. Further research is needed to elucidate the mechanism of this phenomenon.

Our bioinformatics results interestingly also showed that $A L B$ gene expression was significantly lower in ADPKD kidney samples, and this finding was confirmed by qPCR analysis of mRNA expression. ALB is known to act as a carrier protein for a wide range of endogenous molecules including hormones, fatty acids, and metabolites, as well as exogenous drug [60]. The $A L B$ gene has pleiotropic effects of the human serum ALB levels in health and disease, with one study reporting that serum ALB might have a direct role in the inhibition of hepatocellular carcinoma growth either through modulation of $\alpha$-fetoprotein or through its action on growth controlling kinases [61]. Yoo et al. [62] demonstrated that ALB expression is required for adipocyte differentiation of 3T3-L1 cells. Additionally, the ALB gene-encoded preproprotein is proteolytically processed to generate the mature protein, and a peptide derived from this protein, EPI-X4, is an endogenous inhibitor of the CXCR4 chemokine receptor [63]. Our analysis showed that the $A L B$ gene was downregulated by more than 10 -fold in PKD kidney samples compared to control kidney samples, and the mechanism of this alteration and the function of renal ALB protein are unclear in PKD.

A cross-species meta-analysis provided profiling of conserved biological pathways in ADPKD, using data from 9 ADPKD microarray datasets from rats, mice, and humans [23], and their findings supported the possibility of exploring the potential of combination therapy in ADPKD. In our work, although data were only available from 2 gene profiling datasets on clinical samples from ADPKD patients in the GEO database, which greatly limits the effectiveness and scope of the bioinformatics analysis, we identified and validated the downregulation of genes in metabolic pathways, such as amino acid degradation, and this finding may benefit ADPKD research. Additional limitations in our work also include the sample size in GSE35831, which includes data for only 1 normal and 2 ADPKD human renal tissue samples. Additionally, the use of renal samples from ADPKD patients with ESRD may have an impact 
on subsequent analysis, as some changes in gene expression may be attributable to ESRD. Moreover, although mutations in the $P k d 1$ or $P k d 2$ gene are predominantly responsible for ADPKD, the focal and sporadic nature of individual cystogenesis suggests the involvement of another molecular mechanism such as epigenetic alteration [22] or miRNA-mediated regulation [20]. It seems likely that a combinatory and/or serial approach at different stages of disease will be required to more completely explore the mechanism of ADPKD [12].

\section{Conclusion}

The presented bioinformatics analysis of ADPKD gene profiling datasets identified multiple DEGs and enriched signaling pathways in human ADPKD. The abnormal expression of DCN may provide a new therapeutic target for ADPKD. Our results indicate that metabolism regulation and mitochondrion function in ADPKD also may become targets for future research related to therapeutic interventions. Further biological validation of the expression patterns, functions, and mechanisms of the identified DEGs is warranted.

\section{Acknowledgments}

This work was supported by the National Key Research and Development Program of China (2016YFC0901502), National Natural Science Foundation of China (81873595, 81670612), Shanghai Top Priority Key Clinical Disciplines Construction Project (2017ZZ02009).

\section{Disclosure Statement}

The authors declare that there are no conflicts of interest.

The results presented in this paper have not been published previously, in whole or part, except in abstract format.

\section{Author Contributions}

C.M. conceived and coordinated the study. D.L. wrote the paper. C.X. revised the paper. Y.H., D.L., and B.Y. carried out the bioinformatics analysis. L.B., S.S., and L.F. collected the human renal samples. D.L. performed the animal experiment and carried out the real-time PCR, IHC and performed the statistics analysis. S.C. and D.X. performed the zebrafish experiment. All authors reviewed the results and approved the final version of the manuscript.

\section{References}

1 Ong AC, Devuyst O, Knebelmann B, Walz G; ERA-EDTA Working Group for Inherited Kidney Diseases. Autosomal dominant polycystic kidney disease: the changing face of clinical management. Lancet. 2015 May; 385(9981):1993-2002.

2 Sommerer C, Zeier M. Clinical Manifestation and Management of ADPKD in Western Countries. Kidney Dis (Basel). 2016 Oct;2(3):120-7.

3 Xu D, Ma Y, Gu X, Bian R, Lu Y, Xing X, et al. Novel Mutations in the PKD1 and PKD2 Genes of Chinese Patients with Autosomal Dominant Polycystic Kidney Disease. Kidney Blood Press Res. 2018;43(2):297-309.

4 Xue C, Zhou CC, Wu M, Mei CL. The Clinical Manifestation and Management of Autosomal Dominant Polycystic Kidney Disease in China. Kidney Dis (Basel). 2016 Oct;2(3):111-9.

5 Consortium TI; The International Polycystic Kidney Disease Consortium. Polycystic kidney disease: the complete structure of the PKD1 gene and its protein. Cell. 1995 Apr;81(2):289-98. 


\begin{tabular}{l|l}
\hline \multicolumn{2}{l}{ Kidney Blood Press Res 2019;44:533-552 } \\
\hline DOI: 10.1159/000500458 & $\begin{array}{l}\text { (c) 2019 The Author(s). Published by S. Karger AG, Basel } \\
\text { www.karger.com/kbr }\end{array}$ \\
\hline
\end{tabular}

Liu et al.: Bioinformatics Analysis of ADPKD

6 Mochizuki T, Wu G, Hayashi T, Xenophontos SL, Veldhuisen B, Saris JJ, et al. PKD2, a gene for polycystic kidney disease that encodes an integral membrane protein. Science. 1996 May;272(5266):1339-42.

7 Igarashi P, Somlo S. Polycystic kidney disease. J Am Soc Nephrol. 2007 May; 18(5):1371-3.

8 Bagherie-Lachidan M, McNeill H. What drives cyst formation in PKD? J Am Soc Nephrol. 2010 Feb;21(2): 200-2.

9 Harris PC, Torres VE. Polycystic kidney disease. Annu Rev Med. 2009;60(1):321-37.

10 Lanktree MB, Chapman AB. New treatment paradigms for ADPKD: moving towards precision medicine. Nat Rev Nephrol. 2017 Dec;13(12):750-68.

11 Oberdhan D, Cole JC, Krasa HB, Cheng R, Czerwiec FS, Hays RD, et al. Development of the Autosomal Dominant Polycystic Kidney Disease Impact Scale: A New Health-Related Quality-of-Life Instrument. Am J Kidney Dis. 2018 Feb;71(2):225-35.

12 Chang MY, Ong AC. Targeting new cellular disease pathways in autosomal dominant polycystic kidney disease. Nephrol Dial Transplant. 2017 Dec;32(12):2144.

13 Antignac C, Calvet JP, Germino GG, Grantham JJ, Guay-Woodford LM, Harris PC, et al. The Future of Polycystic Kidney Disease Research-As Seen By the 12 Kaplan Awardees. J Am Soc Nephrol. 2015 Sep;26(9):2081-95.

14 Peters DJ, Breuning MH. Autosomal dominant polycystic kidney disease: modification of disease progression. Lancet. 2001 Oct;358(9291):1439-44.

15 Tazón-Vega B, Vilardell M, Pérez-Oller L, Ars E, Ruiz P, Devuyst O, et al. Study of candidate genes affecting the progression of renal disease in autosomal dominant polycystic kidney disease type 1 . Nephrol Dial Transplant. 2007 Jun;22(6):1567-77.

16 Pandey P, Qin S, Ho J, Zhou J, Kreidberg JA. Systems biology approach to identify transcriptome reprogramming and candidate microRNA targets during the progression of polycystic kidney disease. BMC Syst Biol. 2011 Apr; 5(1):56.

17 Menezes LF, Zhou F, Patterson AD, Piontek KB, Krausz KW, Gonzalez FJ, et al. Network analysis of a Pkd1mouse model of autosomal dominant polycystic kidney disease identifies HNF4 $\alpha$ as a disease modifier. PLoS Genet. 2012;8(11):e1003053.

18 Husson H, Manavalan P, Akmaev VR, Russo RJ, Cook B, Richards B, et al. New insights into ADPKD molecular pathways using combination of SAGE and microarray technologies. Genomics. 2004 Sep;84(3):497-510.

19 Menezes LF, Lin CC, Zhou F, Germino GG. Fatty Acid Oxidation is Impaired in An Orthologous Mouse Model of Autosomal Dominant Polycystic Kidney Disease. EBioMedicine. 2016 Jan;5:183-92.

20 Woo YM, Kim DY, Koo NJ, Kim YM, Lee S, Ko JY, et al. Profiling of miRNAs and target genes related to cystogenesis in ADPKD mouse models. Sci Rep. 2017 Oct;7(1):14151.

21 Song X, Di Giovanni V, He N, Wang K, Ingram A, Rosenblum ND, et al. Systems biology of autosomal dominant polycystic kidney disease (ADPKD): computational identification of gene expression pathways and integrated regulatory networks. Hum Mol Genet. 2009 Jul;18(13):2328-43.

22 Woo YM, Bae JB, Oh YH, Lee YG, Lee MJ, Park EY, et al. Genome-wide methylation profiling of ADPKD identified epigenetically regulated genes associated with renal cyst development. Hum Genet. 2014 Mar;133(3):281-97.

23 Chatterjee S, Verma SP, Pandey P. Profiling conserved biological pathways in Autosomal Dominant Polycystic Kidney Disorder (ADPKD) to elucidate key transcriptomic alterations regulating cystogenesis: A cross-species meta-analysis approach. Gene. 2017 Sep;627:434-50.

24 Miryala SK, Anbarasu A, Ramaiah S. Discerning molecular interactions: A comprehensive review on biomolecular interaction databases and network analysis tools. Gene. 2018 Feb;642:84-94.

25 Szklarczyk D, Franceschini A, Wyder S, Forslund K, Heller D, Huerta-Cepas J, et al. STRING v10: protein-protein interaction networks, integrated over the tree of life. Nucleic Acids Res. 2015 Jan;43(Database issue):D44752.

26 Guo Y, Bao Y, Ma M, Yang W. Identification of Key Candidate Genes and Pathways in Colorectal Cancer by Integrated Bioinformatical Analysis. Int J Mol Sci. 2017 Mar;18(4):18.

27 Pathan M, Keerthikumar S, Ang CS, Gangoda L, Quek CY, Williamson NA, et al. FunRich: an open access standalone functional enrichment and interaction network analysis tool. Proteomics. 2015 Aug;15(15):2597-601.

28 Benito-Martin A, Peinado H. FunRich proteomics software analysis, let the fun begin! Proteomics. 2015 Aug; 15(15):2555-6.

29 Gene Ontology C; Gene Ontology Consortium. Gene Ontology Consortium: going forward. Nucleic Acids Res. 2015 Jan;43(Database issue):D1049-56.

30 Ashburner M, Ball CA, Blake JA, Botstein D, Butler H, Cherry JM, et al.; The Gene Ontology Consortium. Gene ontology: tool for the unification of biology. Nat Genet. 2000 May;25(1):25-9.

31 Shannon P, Markiel A, Ozier O, Baliga NS, Wang JT, Ramage D, et al. Cytoscape: a software environment for integrated models of biomolecular interaction networks. Genome Res. 2003 Nov;13(11):2498-504.

32 Saito R, Smoot ME, Ono K, Ruscheinski J, Wang PL, Lotia S, et al. A travel guide to Cytoscape plugins. Nat Methods. 2012 Nov;9(11):1069-76.

33 Kimmel CB, Ballard WW, Kimmel SR, Ullmann B, Schilling TF. Stages of embryonic development of the zebrafish. Dev Dyn. 1995 Jul;203(3):253-310.

34 Sun Z, Amsterdam A, Pazour GJ, Cole DG, Miller MS, Hopkins N. A genetic screen in zebrafish identifies cilia genes as a principal cause of cystic kidney. Development. 2004 Aug;131(16):4085-93.

$35 \mathrm{Xu} \mathrm{D,} \mathrm{Lv} \mathrm{J,} \mathrm{He} \mathrm{L,} \mathrm{Fu} \mathrm{L,} \mathrm{Hu} \mathrm{R,} \mathrm{Cao} \mathrm{Y,} \mathrm{et} \mathrm{al.} \mathrm{Scribble} \mathrm{influences} \mathrm{cyst} \mathrm{formation} \mathrm{in} \mathrm{autosomal-dominant} \mathrm{polycystic}$ kidney disease by regulating Hippo signaling pathway. FASEB J. 2018 Aug;32(8):4394-407. 
Liu et al.: Bioinformatics Analysis of ADPKD

36 Yang B, Chen S, Wu M, Zhang L, Ruan M, Chen X, et al. PHF14: an innate inhibitor against the progression of renal fibrosis following folic acid-induced kidney injury. Sci Rep. 2017 Jan;7(1):39888.

$37 \mathrm{Gu}$ J, Yang M, Qi N, Mei S, Chen J, Song S, et al. Olmesartan Prevents Microalbuminuria in db/db Diabetic Mice Through Inhibition of Angiotensin II/p38/SIRT1-Induced Podocyte Apoptosis. Kidney Blood Press Res. 2016; 41(6):848-64.

38 Agapito G, Guzzi PH, Cannataro M. Visualization of protein interaction networks: problems and solutions. BMC Bioinformatics. 2013;14 Suppl 1:S1.

39 Drummond IA. Polycystins, focal adhesions and extracellular matrix interactions. Biochim Biophys Acta. 2011 Oct;1812(10):1322-6.

40 Okada H, Ban S, Nagao S, Takahashi H, Suzuki H, Neilson EG. Progressive renal fibrosis in murine polycystic kidney disease: an immunohistochemical observation. Kidney Int. 2000 Aug;58(2):587-97.

41 Lee K, Boctor S, Barisoni LM, Gusella GL. Inactivation of integrin- $\beta 1$ prevents the development of polycystic kidney disease after the loss of polycystin-1. J Am Soc Nephrol. 2015 Apr;26(4):888-95.

42 Schaefer L. Small leucine-rich proteoglycans in kidney disease. J Am Soc Nephrol. 2011 Jul;22(7):1200-7.

43 Gubbiotti MA, Vallet SD, Ricard-Blum S, Iozzo RV. Decorin interacting network: A comprehensive analysis of decorin-binding partners and their versatile functions. Matrix Biol. 2016 Sep;55:7-21.

44 Neill T, Schaefer L, Iozzo RV. Decorin as a multivalent therapeutic agent against cancer. Adv Drug Deliv Rev. 2016 Feb;97:174-85.

45 Schaefer L, Gröne HJ, Raslik I, Robenek H, Ugorcakova J, Budny S, et al. Small proteoglycans of normal adult human kidney: distinct expression patterns of decorin, biglycan, fibromodulin, and lumican. Kidney Int. 2000 Oct;58(4):1557-68.

46 Hsieh LT, Nastase MV, Zeng-Brouwers J, Iozzo RV, Schaefer L. Soluble biglycan as a biomarker of inflammatory renal diseases. Int J Biochem Cell Biol. 2014 Sep;54:223-35.

47 Nastase MV, Janicova A, Roedig H, Hsieh LT, Wygrecka M, Schaefer L. Small Leucine-Rich Proteoglycans in Renal Inflammation: Two Sides of the Coin. J Histochem Cytochem. 2018 Apr;66(4):261-72.

48 Rowe I, Chiaravalli M, Mannella V, Ulisse V, Quilici G, Pema M, et al. Defective glucose metabolism in polycystic kidney disease identifies a new therapeutic strategy. Nat Med. 2013 Apr;19(4):488-93.

49 Chiaravalli M, Rowe I, Mannella V, Quilici G, Canu T, Bianchi V, et al. 2-Deoxy-d-Glucose Ameliorates PKD Progression. J Am Soc Nephrol. 2016 Jul;27(7):1958-69.

50 Klawitter J, Klawitter J, McFann K, Pennington AT, Abebe KZ, Brosnahan G, et al. Bioactive lipid mediators in polycystic kidney disease. J Lipid Res. 2014 Jun;55(6):1139-49.

51 Jewell JL, Russell RC, Guan KL. Amino acid signalling upstream of mTOR. Nat Rev Mol Cell Biol. 2013 Mar; 14(3):133-9.

52 Yamamoto J, Nishio S, Hattanda F, Nakazawa D, Kimura T, Sata M, et al. Branched-chain amino acids enhance cyst development in autosomal dominant polycystic kidney disease. Kidney Int. 2017 Aug;92(2):377-87.

53 Klootwijk ED, Reichold M, Helip-Wooley A, Tolaymat A, Broeker C, Robinette SL, et al. Mistargeting of peroxisomal EHHADH and inherited renal Fanconi's syndrome. N Engl J Med. 2014 Jan;370(2):129-38.

54 Raptis V, Loutradis C, Sarafidis PA. Renal injury progression in autosomal dominant polycystic kidney disease: a look beyond the cysts. Nephrol Dial Transplant. 2018 Nov;33(11):1887-95.

$55 \mathrm{Hu}$ XL, Li MP, Song PY, Tang J, Chen XP. AGXT2: an unnegligible aminotransferase in cardiovascular and urinary systems. J Mol Cell Cardiol. 2017 Dec;113:33-8.

56 Klawitter J, Reed-Gitomer BY, McFann K, Pennington A, Klawitter J, Abebe KZ, et al. Endothelial dysfunction and oxidative stress in polycystic kidney disease. Am J Physiol Renal Physiol. 2014 Dec;307(11):F1198-206.

57 Kocyigit I, Yilmaz MI, Orscelik O, Sipahioglu MH, Unal A, Eroglu E, et al. Serum uric acid levels and endothelial dysfunction in patients with autosomal dominant polycystic kidney disease. Nephron Clin Pract. 2013;123(34):157-64.

58 Wang D, Strandgaard S, Borresen ML, Luo Z, Connors SG, Yan Q, et al. Asymmetric dimethylarginine and lipid peroxidation products in early autosomal dominant polycystic kidney disease. Am J Kidney Dis. 2008 Feb; 51(2):184-91.

59 Rodionov RN, Jarzebska N, Weiss N, Lentz SR. AGXT2: a promiscuous aminotransferase. Trends Pharmacol Sci. 2014 Nov;35(11):575-82.

60 Ha CE, Bhagavan NV. Novel insights into the pleiotropic effects of human serum albumin in health and disease. Biochim Biophys Acta. 2013 Dec;1830(12):5486-93.

61 Bağırsakçı E, Şahin E, Atabey N, Erdal E, Guerra V, Carr BI. Role of Albumin in Growth Inhibition in Hepatocellular Carcinoma. Oncology. 2017;93(2):136-42.

62 Yoo W, Lee J, Park S, Kim YS, Lim C, Yoon E, et al. Albumin expression is required for adipocyte differentiation of 3T3-L1 cells. Biochem Biophys Res Commun. 2010 Jun;397(2):170-5.

63 Zirafi O, Hermann PC, Münch J. Proteolytic processing of human serum albumin generates EPI-X4, an endogenous antagonist of CXCR4. J Leukoc Biol. 2016 Jun;99(6):863-8. 